\title{
Analysis of Steady-state Characteristics of Aerodynamic Bearing Based on FLUENT
}

\author{
JIA Chenhui ${ }^{1, a}$, Du Caifeng ${ }^{2, b}$ and QIU Ming ${ }^{3, c}$ \\ Affiliation: School of Mechatronics Engineering, Henan University of Science and Technology; \\ *Address correspondence to this author at Xiyuan Road 48, Box 62, Luoyang, Henan, China, \\ 471003; Tel:0086-13523798226; Email:xjiachenhui@163.com
}

Keywords: Aerodynamic bearing, spherical spiral groove, FLUENT, numerical analysis, bearing capacity.

\begin{abstract}
In order to research the steady-state flow field characteristics of spherical spiral groove aerodynamic bearings, the bearing's lubrication analysis mathematical model is established. Based on CFD technology, and using the FLUENT software of fluid dynamics to analyze the 3D gas film pressure field of the spherical spiral groove aerodynamic bearings. It reveals the laws that effect of the groove width ratio, groove depth ratio, helix angle and groove number under different rotation speed on the steady-state bearing gas film pressure and the bearing capacity, and on this basis the structure of bearing parameters are optimized. The results show that the FLUENT can easily and accurately simulate the complex flow field in the gas film area, and the higher rotation speed, the more obvious gas bearing internal hydrodynamic effect. Therefore, choosing reasonable bearing structural and operating parameters contributes to improve the lubrication performance and bearing capacity.
\end{abstract}

\section{Introduction}

In recent decades, with the development of science and technology, high speed machinery has become an important tool in high efficiency production, and gas bearing plays an important role in many application fields. Gas bearing runs noiseless, low friction, no heat, small vibration and long service life, which is not restricted by the wear [1,2]. Spherical spiral groove aerodynamic bearing is a new type of structure of the journal bearing, it can not only bear radial and axial load, but also has compact structure and high gyration precision, which is widely used in the gyroscope, attitude control device, rotating machinery and other equipment. Either the performance or structure is superior to other types of aerodynamic bearing [3].

At present, the domestic and foreign scholars widely adopt the method of solving the Reynolds equation to research gas film properties of journal bearing. Although the advantage of this method is shortening the solving time, it ignores the inertia force, body-force, axial shear force and circumferential shear force, etc, which can affect the precision of the calculation results. With the development of CFD technology and the improvement of computer performance, the CFD technology can analysis any form of bearing structure. Using the FLUENT to solve its ignoring items, and more accurately reflects the flow field characteristic of the bearing, which can shorten project development cycle [4].

This paper is based on spherical spiral groove aerodynamic bearing as the research object, the bearing's lubrication analysis mathematical model is established. Based on CFD technology, and using the FLUENT software of fluid dynamics, through the laws of the main structural and operating parameters of the groove width ratio, groove depth ratio, helix angle and groove number to analyze the effect to the steady-state bearing gas film pressure distribution and the bearing capacity, and then the structural parameters of the gas bearing design is optimized.

\section{Mathematical Modeling}

Finite Element Model. The spherical spiral groove aerodynamic bearing is mainly composed of rotor and stator, the surface of the rotor is machined a certain number of spiral grooves, and the spiral groove consists of ridge and groove, the larger part of gap called groove and the smaller called ridge, 
the spherical spiral groove aerodynamic bearing section sketch is illustrated in Fig. (1). As the rotor rotates relative to the stator at a high speed, the spiral groove not only forms the staircase effect on bearing, but also increases the pump bearing gas effect, which well promotes aerodynamic bearing hydrodynamic effect [5].

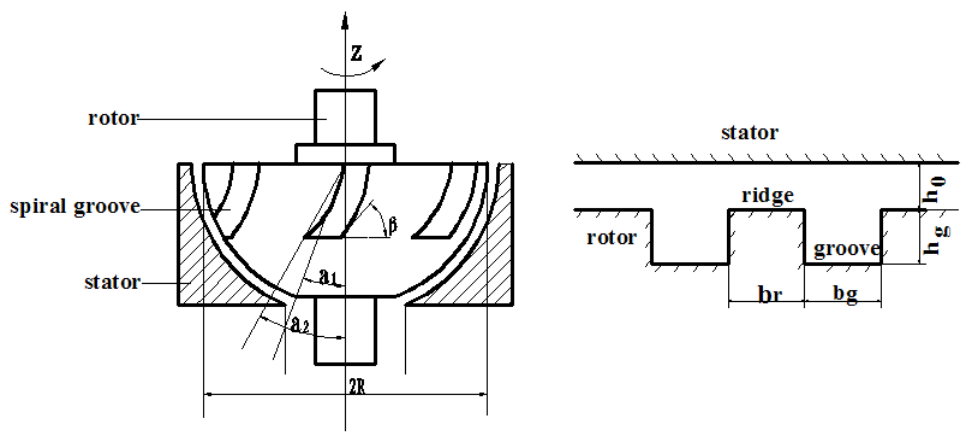

Fig. (1). Schematic diagram of spherical spiral groove aerodynamic bearing

Where $\beta$ is spiral angle, $\mathrm{R}$ is bearing radius, $\omega$ is rotational speed, $b_{r}$ is ridge width, $b_{g}$ is groove width, $h_{g}$ is film thickness at a groove, $h_{0}$ is bearing clearance, $\bar{h}=\left(h_{0}+b_{g}\right) / h_{0}$ is groove depth ratio, $\bar{b}=b_{r} /\left(b_{r}+b_{g}\right)$ is groove width ratio, $\alpha_{1}, \alpha_{2}$ is angle.

The model of the spiral line is established by space spherical coordinates, and referring to [6], the 3D gas film model of the spherical spiral groove aerodynamic bearing is established by the 3D model software Pro/E, the 3D model of the gas film is shown in Fig. (2).

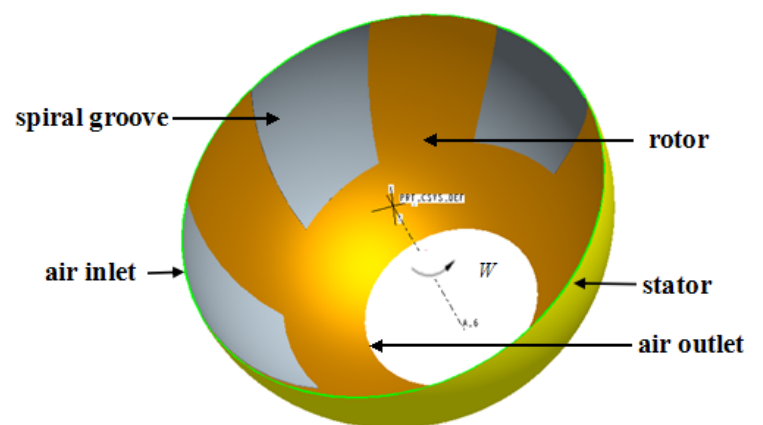

Fig. (2). The 3D model of the gas film of spherical spiral groove aerodynamic bearing

\section{Lubrication Analysis Mathematical Modeling}

Modeling assumption. Spherical spiral groove aerodynamic bearing lubrication analysis mathematical model mainly reflects the gas bearing clearance within the gas film pressure distribution regular. In order to solve the calculation, now do the following assumptions: (1) the lubricating medium is Newton fluid, and gas viscosity coefficient is a constant. (2) the gas within the bearing clearance is in laminar flow state, and the flow direction is the same. (3) no heat exchange between the gas and the wall, no considering the bearing and the thermal deformation of shaft neck in the rotation process. (4) the velocity changes can be ignored on perpendicular to the direction of gas film thickness, the air film pressure along the thickness direction without changing. (5) the gas has no relative sliding in the shaft and bearing surface. (6) assuming the wall is smooth and no considering the influence of wall roughness and slip boundary.

Boundary conditions determine. (1) the big end of the bearing is the air-inlet pressure boundary condition, and the small is the air-outlet pressure boundary condition, the air-inlet and the air-outlet pressure are equal to the atmospheric pressure, namely $p_{0}=1.013 \times 10^{5} \mathrm{~Pa}$. (2) other parts are the wall and no heat exchange, and no relative sliding between the gas and wall. The outer wall is set as a fixed wall, the inner wall is set as a rotating wall, and it is rotational around the bearing eccentric position. 
Mathematical modeling. In order to facilitate the calculation and improve the accuracy of the calculation, by using the FLUENT to simulate the flow field around the rotor of the spiral groove aerodynamic bearing, the main solving is the equation of mass conservation equation, momentum conservation equation [7].

(1) Mass conservation equation

$$
\frac{\partial \rho}{\partial t}+\operatorname{div}(\rho u)=0
$$

Where $\rho$ is the air density; $t$ is the flow time; $\operatorname{div}(u)$ is the divergence of the velocity vector $u$.

(2) The momentum conservation equation

$$
\begin{aligned}
& \frac{\partial(\rho u)}{\partial t}+\operatorname{div}(\rho u \bar{u})=\operatorname{div}(\mu \operatorname{grad} u)-\frac{\partial p}{\partial x}+S_{u} \\
& \frac{\partial(\rho v)}{\partial t}+\operatorname{div}(\rho v \bar{u})=\operatorname{div}(\mu \operatorname{grad} v)-\frac{\partial p}{\partial y}+S_{v} \\
& \frac{\partial(\rho w)}{\partial t}+\operatorname{div}(\rho w \bar{u})=\operatorname{div}(\mu \operatorname{grad} w)-\frac{\partial p}{\partial z}+S_{w}
\end{aligned}
$$

Where $\mu$ is the dynamic viscosity; $S_{u} 、 S_{v} 、 S_{w}$ is momentum conservation equation of the generalized source term; $u 、 v 、 w$ is the velocity vector on the $\mathrm{x}, \mathrm{y}, \mathrm{z}$ direction of the velocity components.

\section{Gas Pressure Distribution}

Calculation and solution. The main design parameters of bearing are as follows: bearing radius $R=4.8 \mathrm{~mm}$; average gas film clearance $h_{0}=10 \mu \mathrm{m}$; gas viscosity $\mu=1.833 \times 10^{-5} \mathrm{pa}$.s ; atmospheric pressure $p_{0}=1.013 \times 10^{5} \mathrm{pa}$; dimensionless eccentricity $\varepsilon=0.3$; dimensionless groove width ratio $\bar{b}=0.5$; dimensionless groove depth ratio $\bar{h}=3.0$; spiral angle $\beta=70^{\circ}$; groove number ; $N g=5$ angle $\alpha_{1}=25^{\circ}, \alpha_{2}=47^{\circ}$.

Adopting the finite volume method to discrete the mass conservation equation and the momentum conservation equation. First, set the iteration parameters, using Linear differential pressure difference format. Other discrete equations use the second-order difference format, and the pressure-velocity coupling uses the SIMPLE algorithm, which is a kind of pressure correction. Due to the continuity equation is not easy to converge in the process of the gas film flow field characteristic analysis, so all equations' calculation of residual error convergence criteria are set to 0.001 , choosing the appropriate owe relaxation factor, then the iterative calculation is starting, and observe the residual curve. When the iterative convergence, modifying the relaxation factor and calculating again, until the results meet the requirements of calculation.

Analysis of gas film pressure distribution. Fig. (3) shows the variation law of static pressure with the different speed of 3D gas film pressure distribution contours.

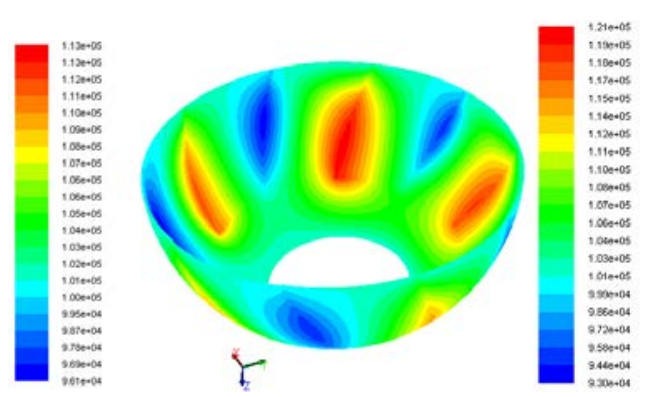

(a) $N=60000 \mathrm{r} / \mathrm{min}$

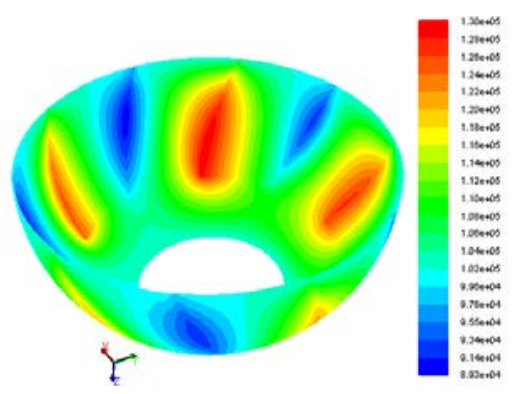

(b) $N=100000 \mathrm{r} / \mathrm{min}$ (c) $N=150000 \mathrm{r} / \mathrm{min}$

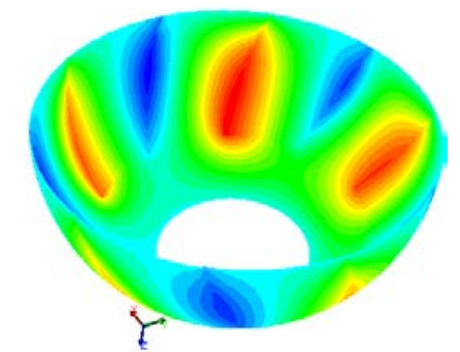

Fig. (3). Gas film pressure contours

From the gas film pressure distribution in Fig. (4), with the increase of rotation speed $N$, the gas 
film pressure of the bearing throughout the circumferential is up from big to small, then from small to large distribution. Because of the existence of five oblique spiral grooves, the gas flow through the region of the groove and the ridge periodically. The gas film pressure curve has five times larger pressure jump in the groove and ridge border, which forms a zigzag distribution in the circumferential direction. The gas film pressure will rise in the convergence field and drop in the diffusion field, and it will reach the maximum in the groove and ridge border. In the region with spiral groove, the gas film pressure and the dynamic pressure effect are more apparent. So the bearing the dynamic pressure effect affected by rotation speed is relatively large, and the aerodynamic bearing hydrodynamic effect is more obvious in the higher speed.

\section{Steady-State Characteristic Factors}

The load capacity is an important index to reflect bearing performance [8]. Fig. (4) shows the variation of the load capacity $W z$ with the change of the groove width ratio $\bar{b}$ under the condition of the different the rotation speed $N$. The bearing capacity increases with the increase of the ratio of groove width $\bar{b}$. When the ratio of groove width $\bar{b}=0.5$, the bearing capacity of three curves is up to the maximum value. If $\bar{b}>0.5$, the load capacity decreases rapidly with the increase of the groove width ratio $\bar{b}$. In this paper, the optimum value of the groove width ratio $\bar{b}$ should be selected 0.5 in the design.

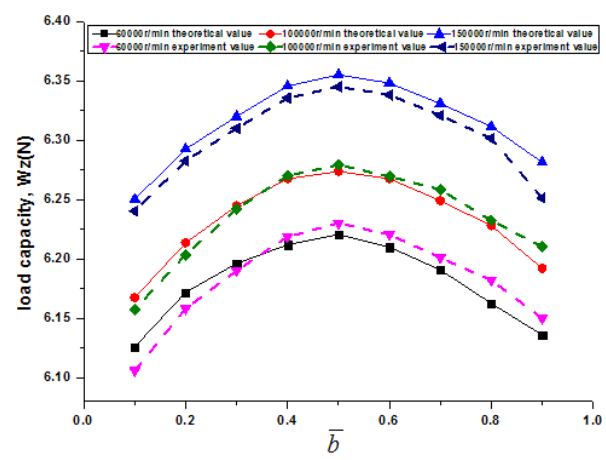

Fig. (4). Variation of load capacity with $\bar{b}$

Fig. (5) shows the variation of the load capacity $W Z$ with the change of the groove depth ratio $\overline{\boldsymbol{h}}$ under the condition of the different the rotation speed $N$. The bearing capacity increases with the increase of the ratio of the groove depth ratio $\bar{h}$. When the groove depth ratio value between 3.0 and 4.0 the three load capacity curves are up to the maximum value. When the groove depth ratio is 3.6, the load capacity is the most suitable for the aerodynamic bearing, and then bearing capacity decreases with the increase of groove depth ratio. That under the same conditions, the smaller groove depth ratio, the better bearing capacity of the bearing, but too small groove depth ratio will bring a certain difficulty to the design and processing, and also cause the different level effect to the stability of the bearing under the dynamic pressure working state. So we should choose the reasonable size of the groove depth ratio.

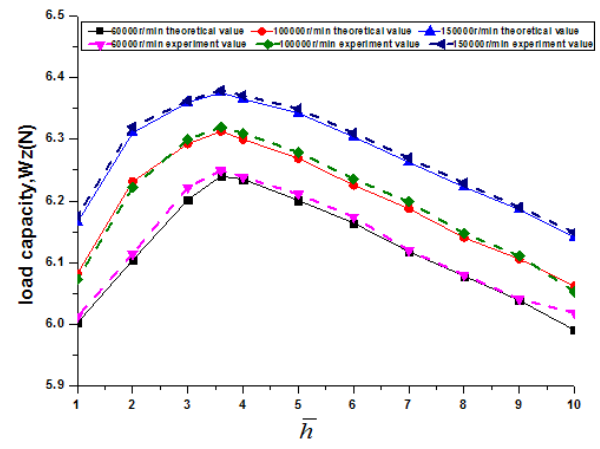

Fig. (5). Variation of load capacity with $\bar{h}$ 
Fig. (6) shows the variation of the load capacity $W z$ with the change of the spiral angle $\beta$ under the condition of the different the rotation speed $N$. The load capacity increases with the increase of the spiral angle. When the load capacity is up to the maximum value between the spiral angle of about $60^{\circ}$ and $80^{\circ}$. The bearing capacity of the three curves reaches maximum value at $70^{\circ}$, the load capacity gradually decreases with the increase of the spiral angle. Due to the spiral angle is an important index to reflect pumping action and the staircase effect, when the spiral angle $\beta$ is $0^{\circ}$, spiral becomes a circle and the spiral groove does not exist, so the staircase effect does not appear. When the spiral angle $\beta$ is $90^{\circ}$, the bearing has been transformed into simple step bearing, the pumping action disappears. Choosing the reasonable spiral angle can improve the load capacity of bearing. In this paper, the optimum value of the spiral angle $\beta$ should be selected $70^{\circ}$ in the design.

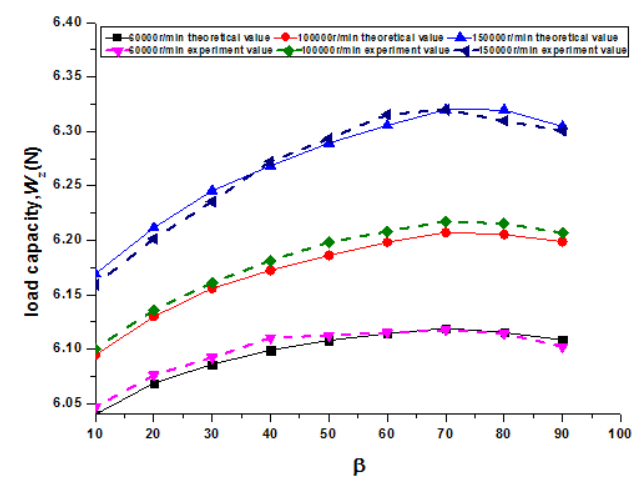

Fig. (6). Variation of load capacity with $\beta$

Fig. (7) shows the variation of the load capacity $W z$ with the change of the number of groove $_{N g}$ under the condition of the different the rotation speed $N$. With the increase of the number of groove $\mathrm{Ng}$, the staircase effect and the load capacity of the bearing will be gradually improved. When the number of groove $\mathrm{Ng}<10$, the load capacity of bearing is more noticeable. When the number of the groove reaches 10 , the load capacity is up to the maximum. When the number of groove $\mathrm{Ng}>10$, the load capacity increasing trend is slow, this is the reason why the more number of groove, the weaker bearing pumping action and staircase effect. At the same time, more number of groove can bring difficulties to the bearing design and processing. It can not increase the number of groove to achieve the purpose of improving the bearing capacity, so choosing the number of groove between 10 and 15 is more reasonable.

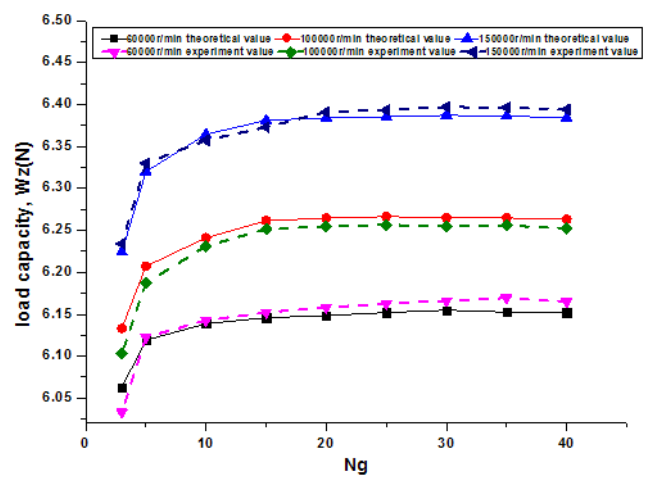

Fig. (7). Variation of load capacity with $\mathrm{Ng}$

In Fig. (4)-(7), the variations of the fitting curve are consistent basically by comparing the theoretical value with the experimental value. The theoretical value coincides with the experiment value, which verifies the correctness of theoretical analysis and calculation.

\section{Conclusions}


1. According to the gas lubrication theory, the bearing's lubrication analysis mathematical model is established, and using fluid dynamics FLUENT software, the FLUENT can easily and accurately simulate the complex flow field in the gas film clearance, and more effectively analyze the gas bearing lubrication performance. In the bearing design, it can effectively shorten the design cycle.

2. With the increase of rotational speed, the gas film pressure of the spherical spiral groove aerodynamic bearing is increasing. Due to the gas bearing has a certain number spiral groove on the spherical surface, the gas film pressure curve has five times larger pressure jump in the groove and ridge border, which forms a zigzag distribution in the circumferential direction, and it reaches the peak value in the groove and ridge border. In the radial direction, the pressure increases inward along the groove, until the groove and ridge border it reaches the peak, and then the gas film pressure reduces inward, until the gas film pressure has reduced to the external environment. Therefore, the existence of the spiral groove can not only promote aerodynamic bearing hydrodynamic effect, but also improve the steady-state load capacity characteristics of the bearing.

3. Under different rotation speed, the bearing structural parameters with groove width ratio, groove depth ratio, groove number and spiral angle have more obvious effect on the bearing capacity. Therefore, a reasonable design parameters should be choosed, when designing the bearing and make the performance of the spiral groove aerodynamic bearing better.

\section{Acknowledgement}

This work is supported by National Natural Science Foundation of China (Grant no: 51475142, 51275155), and Program for Innovative Research Team (in Science and Technology) in University of Henan Province (Grant no: 13IRTSTHN025), and the Education Department Henan Province Science and Technology Rresearch Projects (Grant no: 13A460251).

\section{References}

[1] YunFei Wang: Gas lubricated theory and design of gas bearings, [M]. Beijing: China Machine Press, 1999.

[2] Wei Kang, Jia-zhong Zhang: Stability and bifurcation of symmetrical rotor in self-acting gas journal bearings, [J]. Journal of Aerospace Power, 2007, 22(9): 1537-1543.

[3] Satish C. Sharma, Vikas M. Phalle, S.C. Jain: Performance analysis of a multirecess capillary compensated conical hydrostatic journal bearing, [J]. Tribology International, 2011, 44(5): 617-626.

[4] K.P. Gertzos, P. G. Nikolakopoulos, C. A.Papadopoulos: CFD analysis of journal bearing hydrodynamic lubrication by Bingham Lubricant, [J]. Tribology International, 2008, 41: 1190-1240.

[5] Chen-Hui Jia, Wei Yang, Ming Qiu: Load capacity analysis and structural parameters optimizing of spiral groove aerodynamic bearings, [J]. Journal of Applied Sciences, 2013, 13(18): 3649-3658.

[6] Wangsheng Zhao, Jiangtao Li: Spherical equiangular spiral groove parameter equation modeling and numerically controlled machining, [Z]. Beijing Aerospace Control Instrument Research Institute, 2011.

[7] Wenqi Ma, Fuqiang Liu: Study on the high pressure gas flow field characteristics of spherical bearing, [D]. Dalian Maritime University, 2011. 06.

[8] Zhiwei Lu, Bo Liu, Jun'an Zhang: Research on the wind driven aerodynamic bearings performance, [D]. Xi'an Technological University, 2008. 03. 